\section{Análise da publicidade televisiva de alimentos no contexto das recomendações do Guia Alimentar para a População Brasileira}

\author{
Analysis of TV food advertising in the context of \\ recommendations by the Food Guide for the \\ Brazilian Population
}

\begin{abstract}
Análisis de la publicidad televisiva de alimentos en el contexto de las recomendaciones de la Guía Alimentaria para la Población Brasileña
\end{abstract}

\section{Resumo}

O objetivo do estudo é analisar a publicidade televisiva de alimentos no Brasil com base nas recomendações do Guia Alimentar para a População Brasileira de 2014. A programação dos quatro canais mais populares de televisão foi gravada em janeiro de 2014, por dois dias não consecutivos. Os comerciais foram categorizados em (i) alimentos e bebidas, (ii) restaurantes e (iii) serviços, bens e produtos não alimentícios, sendo aqueles referentes à alimentação subdivididos conforme recomendações do guia. As categorias de comerciais foram descritas por meio de sua frequência (e intervalo de 95\% de confiança), para o conjunto total dos registros e segundo o dia da semana. Dos 2.732 comerciais que foram identificados, a publicidade de alimentos e bebidas representou a terceira maior categoria anunciada, com 10,2\%. Nessa categoria, os alimentos ultraprocessados corresponderam a 60,7\% dos anúncios, e os alimentos in natura ou minimamente processados a cerca de $7 \%$. A realidade evidenciada se opõe às recomendações do guia, reforçando a importância de ações para a regulamentação da publicidade de alimentos no Brasil.

Publicidade de Alimentos; Televisão; Doença Crônica
Emanuella Gomes Maia 1

Bruna Vieira de Lima Costa 1

Francielly de Souza Coelho 1

Julia Soares Guimarães 1

Rafaela Garcia Fortaleza 1

Rafael Moreira Claro 1

doi: 10.1590/0102-311X00209115

\section{Correspondência}

E. G. Maia

Universidade Federal de Minas Gerais.

Av. Prof Alfredo Balena 190, Belo Horizonte, MG

30130-100, Brasil.

manugmaia@hotmail.com

1 Universidade Federal de Minas Gerais, Belo Horizonte, Brasil. 


\section{Introdução}

Desde a segunda metade do século XX, o perfil de alimentação e de prática de atividade física dos brasileiros tem modificado intensamente 1,2,3, caracterizando-se pelo aumento do consumo de alimentos ultraprocessados 1,3,4,5 e adoção de um estilo de vida sedentário ${ }^{2}$. Nesse cenário, destaca-se o hábito de assistir à televisão ou o uso de jogos eletrônicos pela população, que por sua vez, não só inibe a realização de atividades físicas como também expõe o indivíduo a um volume cada vez maior de publicidade de alimentos 6,7,8. No Brasil, quase toda população (99\%) possui ao menos um aparelho de televisão em casa (Instituto Brasileiro de Opinião Pública e Estatística. Media book, 2013. http:// www.mediabook.ibope.com/, acessado em 30/Nov/2015), e o hábito de assistir à televisão por períodos prolongados é referido por cerca de 1 a cada 4 adultos $\left(28,9 \%, \geq 3\right.$ horas/dia) ${ }^{9}$ e 3 a cada 4 escolares

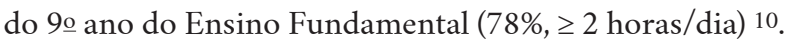

Evidências associam o hábito de assistir à televisão por períodos prolongados com o aumento do risco de desenvolvimento tanto de obesidade 11,12,13,14,15 quanto de outras doenças crônicas não transmissíveis (DCNT), como hipertensão arterial, doenças cardiovasculares e diabetes 16,17,18,19,20. Parte dessa relação se deve à exposição do indivíduo à publicidade de alimentos e bebidas que, por sua vez, incentiva o consumo alimentar inadequado 21 . Tanto em países desenvolvidos quanto naqueles em desenvolvimento, grande parte da publicidade televisiva de alimentos e bebidas está relacionada a produtos com alto teor de gorduras, açúcares e sódio, sendo praticamente inexistentes anúncios de alimentos saudáveis, como grãos integrais, frutas e hortaliças 8,15,22,23.

No Brasil, diversos estudos realizados nos últimos 15 anos evidenciam cenário semelhante $22,24,25,26$. Verifica-se não apenas a baixa qualidade nutricional dos produtos anunciados - geralmente de alto valor calórico e contendo elevada quantidade de gorduras, açúcares e sódio - bem como a utilização de estratégias publicitárias destinadas a explorar populações mais vulneráveis - como o uso de personagens de desenhos animados e anúncios destinados ao público infantil 22,24,25,26.

Com o intuito de promover a alimentação saudável pela população e contrapor o cenário atual, o Ministério da Saúde publicou a segunda edição do Guia Alimentar para a População Brasileira 27. Esse documento (doravante denominado simplesmente "Guia") aborda os princípios e as recomendações de uma alimentação adequada e saudável, configurando-se como instrumento de apoio às ações de educação alimentar e nutricional. Entre as suas recomendações, o Guia prioriza o consumo de alimentos frescos (in natura ou minimamente processados) e preparações culinárias em detrimento ao consumo de alimentos ultraprocessados. No intuito de auxiliar os indivíduos no atendimento dessas recomendações, o Guia destaca a necessidade do pensamento crítico quanto à informação disponível sobre a alimentação, principalmente no que diz respeito à publicidade de alimentos e bebidas. Contudo, poucos estudos se dedicaram a abordar a publicidade de alimentos e bebidas nos canais de televisão aberta do país em um contexto propriamente nutricional 8,22, inviabilizando, até o presente momento, verificar a adequação desta publicidade às recomendações do Guia.

Desse modo, este trabalho tem por objetivo analisar a publicidade televisiva de alimentos nos canais de televisão aberta do país, no contexto das recomendações do Guia Alimentar para a População Brasileira de 2014.

\section{Métodos}

\section{Coleta e organização dos dados}

A programação dos quatro canais de televisão aberta de maior audiência no Brasil (Instituto Brasileiro de Opinião Pública e Estatística. Evolução anual de audiência - TV - PNT, 2013. http://www. ibope.com.br, acessado em 30/Nov/2015) foi analisada neste estudo. Realizou-se a gravação digital (armazenada em meio eletrônico por intermédio de um sintonizador digital de TV) da programação dos canais selecionados, simultaneamente, em janeiro de 2014. Foram registrados dois dias de programação, não consecutivos, sendo um dia de semana (entre segunda-feira e sexta-feira) e outro de final de semana (sábado ou domingo), selecionados ao acaso, permitindo captar a variabilidade da 
programação. Nos dias sorteados a gravação ocorreu em um período sequencial de 16,5 horas, tendo início às $6 \mathrm{~h} 30 \mathrm{~min}$ e término às $23 \mathrm{~h} 00 \mathrm{~min}$.

Em cada um dos intervalos comerciais das horas de programação registradas foram identificados os tipos de produtos anunciados, classificando-os entre três categorias: (i) alimentos e bebidas; (ii) restaurantes; (iii) serviços, bens e produtos não alimentícios. Em seguida, os anúncios de alimentos foram classificados de acordo com as diretrizes do Guia (contidas no Capítulo 2, A Escolha dos Alimentos). Baseado na natureza, intensidade e propósito do processamento dos alimentos, o Guia sugere quatro grupos: alimentos in natura ou minimamente processados; ingredientes culinários; alimentos processados; e alimentos ultraprocessados. Do mesmo modo, os anúncios de restaurantes foram classificados como estabelecimentos tradicionais ou do tipo fast food (conforme recomendação contida no Capítulo 4, O Ato de Comer e a Comensalidade). Por fim, os demais anúncios (não relacionados aos alimentos) foram classificados entre 15 categorias de serviços, bens e produtos não alimentícios: programação da emissora; cosméticos e produtos de higiene; bancos, lotéricas e produtos financeiros; medicamentos, suplementos e aparelhos/próteses; serviço de telecomunicação; eventos e divulgação; instituições educacionais; automóveis e revendedoras; entidades públicas e programas governamentais; lojas de varejo, móveis e eletroeletrônicos; papelarias, livrarias e material escolar; materiais de limpeza; lojas de vestuário e acessórios; serviços de vendas on-line e entrega; outros serviços e produtos.

\section{Análise dos dados}

O conjunto completo dos intervalos comerciais registrados foi inicialmente descrito por meio da frequência absoluta, relativa e seu intervalo de 95\% de confiança (IC95\%) de cada uma das categorias analisadas: alimentos e bebidas; restaurantes; serviços, bens e produtos não alimentícios. Em seguida, os subconjuntos de anúncios classificados na categoria de "alimentos e bebidas" foram descritos considerando-se os grupos de alimentos (in natura ou minimante processados; ingredientes culinários; processados; e ultraprocessados) e os de "restaurantes", levando-se em conta os tipos de estabelecimentos comerciais (restaurantes tradicionais ou do tipo fast food) elencados entre as diretrizes do Guia. A análise dos subconjuntos da categoria "alimentos e bebidas" foi realizada para o conjunto total das gravações e estratificada segundo dia da semana em que se realizou a gravação (dia da semana ou fim de semana).

O teste de qui-quadrado foi utilizado para a identificação de diferenças estatisticamente significativas na frequência de cada categoria de alimento entre dia da semana e fim de semana.

O software estatístico Stata, versão 12.1 (StataCorp LP, College Station, Estados Unidos), foi usado para organização e análise dos dados. A significância foi determinada em $\mathrm{p}<0,05$.

\section{Resultados}

Um total de 7.920 minutos de programação foi registrado (132 horas), permitindo identificar 2.754 anúncios publicitários (20,7 anúncios por hora ou aproximadamente 1 a cada 3 minutos).

A publicidade de alimentos e bebidas e restaurantes respondeu por pouco mais de um a cada dez anúncios registrados (10,2\% para alimentos e bebidas e 0,9\% para restaurantes) (Tabela 1). Os anúncios de alimentos e bebidas representaram a terceira categoria de produtos mais anunciados (10,2\%), inferior apenas à frequência de anúncios relacionados à programação da própria emissora $(21,7 \%)$ e aos cosméticos e produtos de higiene $(10,7 \%)(\mathrm{p}<0,05)$ (Tabela 1). Quanto à frequência de propagandas relacionadas aos restaurantes, não foram observadas diferenças significativas entre aquelas do tipo tradicional e as do tipo fast food ( $\mathrm{p}>0,05)$ (Tabela 1$)$.

Dentro da categoria de alimentos e bebidas, não observou-se a publicidade de produtos processados. Já os produtos ultraprocessados responderam pela ampla maioria dos anúncios, tendo participação aproximada de 60\% (Tabela 2). Os anúncios referentes a esse tipo de alimento superaram em mais de oito vezes os de alimentos in natura ou minimamente processados $(7,4 \%)$ e em cerca de duas vezes aqueles de bebidas alcoólicas (31,9\%). Entre os alimentos ultraprocessados mais frequentemente anunciados destacam-se as bebidas adoçadas (31,6\%), os sorvetes, chocolates e outros doces (17\%) e 
Tabela 1

Frequência absoluta e relativa de anúncios publicitários em quatro canais de televisão aberta, segundo categorias de produtos, bens ou serviços. Brasil, 2014.

\begin{tabular}{|c|c|c|c|}
\hline Categoria de produtos, bens ou serviços & $\mathbf{n}$ & $\%$ & $\mathrm{IC} 95 \%$ \\
\hline Alimentos e bebidas & 282 & 10,20 & $9,10-11,40$ \\
\hline In natura ou minimamente processados e ingredientes culinários & 21 & 0,76 & $0,68-0,85$ \\
\hline Alimentos ultraprocessados & 171 & 6,19 & $5,52-6,91$ \\
\hline Bebidas alcoólicas & 90 & 3,25 & $2,90-3,64$ \\
\hline Restaurantes & 22 & 0,90 & $0,71-1,50$ \\
\hline Restaurantes tradicionais & 12 & 0,50 & $0,39-0,80$ \\
\hline Restaurantes fast food & 10 & 0,40 & $0,32-0,70$ \\
\hline Serviços, bens e produtos não alimentícios & 2.450 & 88,90 & $88,20-90,70$ \\
\hline Programação da emissora & 597 & 21,66 & $21,49-22,10$ \\
\hline Cosméticos e produtos de higiene & 294 & 10,67 & $10,58-10,88$ \\
\hline Bancos, financeiras e lotéricas & 166 & 6,02 & $5,98-6,15$ \\
\hline Medicamentos, suplementos e aparelhos/próteses & 147 & 5,33 & $5,29-5,44$ \\
\hline Serviço de telecomunicação & 128 & 4,64 & $4,61-4,74$ \\
\hline Eventos e divulgação & 127 & 4,61 & $4,57-4,70$ \\
\hline Instituições educacionais & 121 & 4,39 & $4,36-4,48$ \\
\hline Automóveis e revendedoras & 107 & 3,88 & $3,85-3,96$ \\
\hline Entidades públicas e programas governamentais & 80 & 2,90 & $2,88-2,96$ \\
\hline Lojas de varejo, móveis e eletroeletrônicos & 80 & 2,90 & $2,88-2,96$ \\
\hline Papelarias, livrarias e material escolar & 72 & 2,61 & $2,59-2,67$ \\
\hline Materiais de limpeza & 71 & 2,58 & $2,56-2,63$ \\
\hline Lojas de vestuário e acessórios & 68 & 2,47 & $2,45-2,52$ \\
\hline Serviços de vendas on-line e entrega & 61 & 2,21 & $2,20-2,26$ \\
\hline Outros & 331 & 12,01 & $11,92-12,25$ \\
\hline Total & 2.754 & 100,00 & \\
\hline
\end{tabular}

IC95\%: intervalo de 95\% de confiança.

os cereais matinais (5,3\%). A baixa frequência de anúncios de alimentos in natura ou minimamente processados e de ingredientes culinários é um impeditivo para a divisão desse conjunto de alimentos em subgrupos. No entanto, dentre esses anúncios, nenhum relacionado a frutas e hortaliças foi verificado (dados não mostrados).

Quando comparados os anúncios registrados em dias de semana com aqueles veiculados durante o fim de semana, observou-se que não houve diferença significativa na frequência e distribuição dos mesmos (Tabela 2).

\section{Discussão}

Este é o primeiro estudo da publicidade televisiva no Brasil a considerar as recomendações do Guia Alimentar para a População Brasileira de 2014. A análise de mais de 130 horas de programação televisiva dos quatro principais canais de televisão aberta do país propiciou uma compreensão detalhada acerca do tipo de alimento anunciado e sua adequação às recomendações do Guia. Ainda que a participação dos anúncios de alimentos e bebidas possa parecer pouco expressiva (10,2\%), ela representa a terceira maior categoria de todos os produtos anunciados, atrás somente de anúncios da programação da própria emissora $(21,7 \%)$ e de cosméticos e produtos de higiene (10,7\%). Adicionalmente, das propagandas referentes aos alimentos e bebidas, destacam-se os alimentos ultraprocessados $(60,7 \%)$ e bebidas alcoólicas (31,9\%), que por sua vez se opõem às recomendações do Guia. 


\section{Tabela 2}

Frequência absoluta e relativa de anúncios publicitários de alimentos e bebidas, durante a semana e aos finais de semana, em 4 canais de televisão aberta, segundo categorias baseadas nas recomendações do Guia Alimentar para a População Brasileira. Brasil, 2014.

\begin{tabular}{|c|c|c|c|}
\hline Grupo e subgrupo de alimentos & $\mathbf{n}$ & $\%$ & $\mathrm{IC95 \%}$ \\
\hline \multicolumn{4}{|l|}{ Total } \\
\hline In natura ou minimamente processados e ingredientes culinários & 21 & 7,40 & $3,50-11,40$ \\
\hline Ultraprocessados & 171 & 60,70 & $58,20-63,10$ \\
\hline Bebidas adoçadas & 89 & 31,59 & $30,29-32,84$ \\
\hline Refrigerantes e sucos artificiais & 64 & 22,72 & $21,78-23,62$ \\
\hline Bebidas lácteas & 25 & 8,87 & $8,51-9,23$ \\
\hline Sorvete, chocolate e outros doces & 48 & 17,04 & $16,34-17,71$ \\
\hline Cereais matinais & 15 & 5,32 & $5,11-5,54$ \\
\hline Refeições prontas e misturas industrializadas & 13 & 4,62 & $4,42-4,80$ \\
\hline Molhos e condimentos & 6 & 2,13 & $2,04-2,21$ \\
\hline Bebidas alcoólicas & 90 & 31,90 & $26,40-37,40$ \\
\hline \multicolumn{4}{|l|}{ Dia de semana } \\
\hline In natura ou minimamente processados e ingredientes culinários & 10 & 6,90 & $2,70-11,10$ \\
\hline Ultraprocessados & 90 & 62,10 & $58,60-65,50$ \\
\hline Bebidas adoçadas & 44 & 30,36 & $28,65-32,02$ \\
\hline Refrigerantes e sucos artificiais & 31 & 21,39 & $20,18-22,56$ \\
\hline Bebidas lácteas & 13 & 8,97 & $8,46-9,46$ \\
\hline Sorvete, chocolate e outros doces & 24 & 16,56 & $15,63-17,47$ \\
\hline Cereais matinais & 11 & 7,59 & $7,16-8,01$ \\
\hline Refeições prontas e misturas industrializadas & 6 & 4,14 & $3,91-4,37$ \\
\hline Molhos e condimentos & 5 & 3,45 & $3,26-3,64$ \\
\hline Bebidas alcoólicas & 45 & 31,00 & $23,40-38,70$ \\
\hline \multicolumn{4}{|l|}{ Final de semana } \\
\hline In natura ou minimamente processados e ingredientes culinários & 11 & 8,03 & $1,80-14,30$ \\
\hline Ultraprocessados & 81 & 59,17 & $55,80-62,50$ \\
\hline Bebidas adoçadas & 45 & 32,89 & $31,00-34,72$ \\
\hline Refrigerantes e sucos artificiais & 33 & 24,12 & $22,73-25,46$ \\
\hline Bebidas lácteas & 12 & 8,77 & $8,27-9,26$ \\
\hline Sorvete, chocolate e outros doces & 24 & 17,52 & $16,53-18,52$ \\
\hline Cereais matinais & 4 & 2,92 & $2,76-3,09$ \\
\hline Refeições prontas e misturas industrializadas & 7 & 5,11 & $4,82-5,40$ \\
\hline Molhos e condimentos & 1 & 0,73 & $0,69-0,77$ \\
\hline Bebidas alcoólicas & 45 & 32,80 & $24,90-40,80$ \\
\hline
\end{tabular}

IC95\%: intervalo de 95\% de confiança.

Potenciais limitações devem ser observadas na apreciação dos resultados do presente trabalho. Dentre essas, destacam-se: (i) a não inclusão de todas as emissoras de televisão aberta do Brasil; (ii) o curto período de tempo para a coleta dos dados em cada emissora (dois dias); (iii) a restrição da coleta a um único mês; e (iv) a não possibilidade de estratificação dos resultados segundo a idade do público. Contudo, acredita-se que nenhuma dessas questões comprometa a validade dos resultados apresentados. A definição a respeito do número de emissoras e de dias a serem registrados baseou-se nas recomendações internacionais para o monitoramento de publicidade utilizada pela Rede INFORMAS (International Network for Food and Obesity/Non-communicable Diseases Research, Monitoring and Action Support) 28, levando-se em conta dados sobre a audiência dos canais disponibilizados pelo Instituto Brasileiro de Opinião Pública e Estatística (IBOPE. Evolução anual de audiência - TV - PNT, 2013. http://www.ibope.com.br, acessado em 30/Nov/2015). Além disso, dados de estudos 
longitudinais, conduzidos em países desenvolvidos, indicando a baixa influência sazonal na quantidade de anúncios de alimentos e na qualidade dos produtos anunciados 29 subsidiaram a restrição da coleta dos dados a um único período do ano. Por fim, a ausência de medição da audiência segmentada por idade e o fato de que a maior parte da programação da TV aberta é classificada como "aberta a todas as faixas etárias” inviabiliza a realização de análises estratificadas segundo idade do público preferencial. De todo modo, foi possível identificar comerciais veiculados em programas infantis (mais facilmente identificáveis na grade de programação das emissoras). Ao todo, 10\% dos anúncios foram veiculados durante a programação infantil e a distribuição desses entre alimentos e bebidas; restaurantes; e serviços, bens e produtos não alimentícios foi semelhante à observada para o conjunto completo da amostra estudada (assim como para os anúncios veiculados em programas não infantis - dados não mostrados). Todavia, a baixa frequência de anúncios de alimentos e bebidas na programação infantil não permitiu identificar (com precisão satisfatória) a divisão desses anúncios entre as categorias do Guia.

Valor semelhante ao apresentado no presente trabalho para a participação de alimentos e bebidas no total de anúncios no Brasil (10,2\%) já foi identificado em um estudo publicado em 2010 30. Naquela ocasião, em estudo multicêntrico sobre publicidade de alimentos, identificou-se a participação de 18\% dos alimentos e bebidas no total de anúncios das principais emissoras do país, destacando o Brasil como o país com a menor taxa de participação de alimentos e bebidas (11\%) dentre os 11 países analisados no trabalho ${ }^{30}$. Quando comparados à participação de 30,2\% de alimentos e bebidas (1.696 anúncios), estimada em um estudo que analisou a programação das três principais redes de televisão aberta do país entre agosto de 1998 e março de 2000 22, os valores mais recentemente estimados próximos de 10\% (282 anúncios) - podem significar que a publicidade de alimentos e bebidas vem perdendo espaço no país.

É consenso na literatura que a maior parte dos anúncios comerciais de alimentos e bebidas veiculados na televisão se refere a alimentos de baixo valor nutricional. Em um trabalho realizado na Alemanha, com a análise de 16.062 anúncios publicitários conduzidos em dez emissoras de televisão, entre $06 \mathrm{~h} 00 \mathrm{~min}$ e $22 \mathrm{~h} 00 \mathrm{~min}$, a publicidade de alimentos correspondeu a 19,9\% do total de propagandas (3.197 anúncios), sendo 73\% (2.334 anúncios) relacionados a produtos de má qualidade nutricional (ultraprocessados) 31. Outro estudo feito na Turquia que analisou os quatro canais abertos mais populares, das $08 \mathrm{~h} 00 \mathrm{~min}$ às $24 \mathrm{~h} 00 \mathrm{~min}$, durante quatro dias (dois dias de semana e dois de fim de semana), chegou a resultados semelhantes, com 32,1\% dos anúncios registrados relacionados à alimentação (2.848 anúncios), sendo $81 \%$ dos comerciais exibidos referentes a produtos de alta densidade energética e alto teor de gordura e açúcar 32. Da mesma forma, em um estudo brasileiro desenvolvido em 2009, que analisou anúncios de três emissoras, das 08h00min às $18 \mathrm{~h} 00 \mathrm{~min}$, durante dez dias de semana e finais de semana, dos 1.369 anúncios identificados, 13,8\% (189 anúncios) se referiam a alimentos, sendo que os grupos "açúcares e doces" e "óleos, gorduras e sementes oleaginosas" prevaleceram com $48,1 \%$ e $29,1 \%$, respectivamente ${ }^{8}$. Em nosso trabalho, cerca de $60 \%$ dos comerciais de alimentos e bebidas referiam a grupo de alimentos processados e ultraprocessados, destacando-se aqueles ricos em açúcar, "refrigerantes e sucos artificiais" (22,7\%) e "sorvetes, chocolate e outros doces" (17\%).

A exposição à publicidade de alimentos ultraprocessados, como aquela relatada em mais de $60 \%$ dos anúncios de alimentos e bebidas analisados no presente estudo, é considerada um determinante central do aumento global da obesidade nas últimas décadas 16,33,34. Com isso, torna-se urgente a adoção de medidas específicas destinadas à regulação da publicidade de alimentos no país. Cabe destacar que medidas dessa natureza já foram testadas, sem sucesso, em um passado recente. A Resolução RDC no 24/2010 35, da Agência Nacional de Vigilância Sanitária, exige que qualquer anúncio de alimentos com conteúdo excessivo de açúcar, sódio, gordura saturada ou trans seja acompanhado de advertências sobre os prejuízos que o consumo em grandes quantidades desses alimentos podem trazer à saúde. A publicação dessa resolução encontrou forte reação por parte das associações que representam as indústrias de alimentos e o setor de empresas de comunicação e publicidade 36, resultando numa intensa disputa judicial que terminou por inviabilizar a aplicação da RDC. Atualmente, ainda não existe um regulamento específico para a publicidade de alimentos e bebidas, entretanto, segundo o Código de Defesa do Consumidor (Lei Federal no 8.078/1990) 37, é proibida qualquer publicidade enganosa ou abusiva que seja capaz de induzir o consumidor a se comportar de forma prejudicial ou perigosa à saúde. Para o público infantil, essa proteção é reforçada pelo posicionamento do Conselho 
Nacional de Defesa dos Direitos da Criança e do Adolescente (CONANDA), por meio da Resolução no 163/2014 38, assim como pelo recente entendimento do Superior Tribunal de Justiça (STJ) de que a publicidade quando destinada às crianças é ilegal e abusiva 39 .

As principais críticas à regulação da publicidade de alimentos são que essa medida intervém no direito de livre escolha dos cidadãos; que a publicidade não determina a quantidade de consumo, sendo esta a responsável pelos malefícios do consumo desses alimentos; e que não há evidências de que essa medida reduziria a obesidade, uma vez que ela é multifatorial. Além disso, quanto às propagandas destinadas ao público infantil, as críticas referem-se ao fato de que a determinação e controle da dieta das crianças são responsabilidade dos pais; que é essa publicidade que financia a programação infantil e sua proibição levaria ao cancelamento de diversos programas; que o fato de não haver a publicidade de um alimento não impedirá que a criança o consuma 36 . Mas essas críticas podem ser facilmente combatidas.

É fato que todos os cidadãos têm o direito de livre escolha e com isso podem consumir o que desejam. Porém é dever do Estado zelar pela sua saúde e bem-estar e protegê-los para que não sejam enganados e manipulados, de forma a consumirem determinados produtos não saudáveis. Além disso, diversos estudos comprovam que a exposição à publicidade desperta o interesse no consumo do produto anunciado $23,40,41,42$, sendo essa a razão fundamental da publicidade. No que tange especificamente à publicidade de alimentos não saudáveis, todo corpo de evidência disponível até o momento aponta para a sua associação ao desejo de consumi-los, tanto entre adultos 40,41 como entre crianças 8,42,43. Ainda, de forma semelhante, evidências indicam também que a presença mais frequente da publicidade de alimentos saudáveis estimularia o consumo desses produtos $43,44,45$.

Com relação especificamente às crianças, de fato é dever dos pais a educação dos filhos, incluindo a educação alimentar. Porém, eles não estão sozinhos nessa tarefa, contando com a participação das escolas e do meio em que as crianças estão inseridas. Quando esse meio é um ambiente que estimula hábitos não saudáveis (como a influência da publicidade no desejo de consumo das crianças), torna-se complicada e quase impossível a tarefa dos pais de determinarem e controlarem a dieta dos filhos. A regulação da publicidade vem como um facilitador para que os pais possam educar seus filhos com menos interferências. Somado a isso, os resultados do presente estudo e de outros mostram que no Brasil a quantidade de propagandas de alimentos e bebidas na televisão aberta é muito menor que a de serviços, bens e produtos não alimentícios 8,22, logo, não é possível afirmar que é a publicidade de alimentos que sustenta os programas.

O consumo de alimentos ocorre porque há um estímulo, seja ele por necessidades fisiológicas ou ambientais. Mas o que faz com que uma criança escolha um determinado produto em relação aos outros semelhantes é o poder de persuasão da publicidade. Isso porque a criança não tem conhecimento suficiente para distinguir as características nutricionais de um alimento e saber se ele é bom ou não. Mas, influenciada pela propaganda e pelos apelos visuais do produto, ela acredita que ele seja melhor que os demais 46 . Assim, a proibição da publicidade de alimentos não saudáveis diminuiria a preferência das crianças por esses produtos. Um comercial realmente não determina a quantidade que a criança consome, porém se a propaganda desperta o interesse pelo produto, a repetição do anúncio gera a repetição do desejo. Por conseguinte, a criança não consome apenas uma vez o alimento, mas enquanto houver aquela propaganda haverá o anseio pelo produto.

Por fim, um estudo conduzido nos Estados Unidos mostrou que a proibição da publicidade de alimentos não saudáveis diminuiria o consumo calórico e, com isso, a obesidade e o sobrepeso em crianças 47, combatendo as críticas e reforçando a necessidade da regulação da publicidade de alimentos.

\section{Conclusão}

Os resultados desse trabalho mostraram a importante participação da veiculação de alimentos e bebidas na publicidade televisiva no país, que fica atrás somente de anúncios da própria programação das emissoras. A maior parcela dessa publicidade faz referência a alimentos ultraprocessados, especialmente os ricos em açúcar. Essa realidade se opõe às recomendações do Guia Alimentar para a População Brasileira, reforçando a importância de ações de regulamentação da publicidade televisiva de alimentos no Brasil. 


\section{Colaboradores}

E. G. Maia e R. M. Claro participaram da concepção e delineamento do trabalho, coleta e análise dos dados, redação e revisão do artigo. B. V. L. Costa colaborou na análise dos dados, redação e revisão do artigo. F. S. Coelho, J. S. Guimarães e R. G. Fortaleza contribuíram na coleta de dados e redação do artigo. Todos os autores aprovaram a versão final.

\section{Agradecimentos}

Os autores agradecem à Coordenação de Aperfeiçoamento de Pessoal de Nível Superior (Capes), ao Conselho Nacional de Desenvolvimento Científico e Tecnológico $(\mathrm{CNPq})$ e à Pró-Reitoria de Pesquisa da Universidade Federal de Minas Gerais (PRPQ/ UFMG) pelo financiamento de bolsa de estudo (Mestrado, PIBIC e PIBIC/AF).

\section{Referências}

1. Monteiro CA, Levy RB, Claro RM, Castro IRR, Cannon G. A new classification of foods based on the extent and purpose of their processing. Cad Saúde Pública 2010; 26:2039-49.

2. Chaput JP, Klingenberg L, Astrup A, Sjödin AM. Modern sedentary activities promote overconsumption of food in our current obesogenic environment. Obes Rev 2011; 12:12-20.

3. Martins APB, Levy RB, Claro RM, Moubarac JC, Monteiro CA. Participação crescente de produtos ultraprocessados na dieta brasileira (1987-2009). Rev Saúde Pública 2013; 47: 656-65.

4. Canella DS, Levy RB, Martins APB, Claro RM, Moubarac JC, Baraldi LG, et al. Ultraprocessed food products and obesity in Brazilian households (2008-2009). PLoS One 2014; 9:e92752.

5. Louzada MLC, Martins APB, Canella DS, Baraldi LG, Levy RB, Claro RM, et al. Ultraprocessed foods and the nutritional dietary profile in Brazil. Rev Saúde Pública 2015; 49:38.

6. Tudor-Locke C, Craig C, Cameron C, Griffiths JM. Canadian children's and youth's pedometer-determined steps/day, parent-reported TV watching time, and overweight/obesity: The CANPLAY Surveillance Study. Int J Behav Nutr Phys Act 2011; 8:66.

7. Lazarou C, Kalavana T. Urbanization influences dietary habits of Cypriot children: the CYKIDS study. Int J Public Health 2009; 54:69-77.

8. Costa SMM, Horta PM, Santos LC. Análise dos alimentos anunciados durante a programação infantil em emissoras de canal aberto no Brasil. Rev Bras Epidemiol 2013; 16:976-83.

9. Instituto Brasileiro de Geografia e Estatística. Pesquisa Nacional de Saúde 2013: percepção do estado de saúde, estilos de vida e doenças crônicas. v. 1. Rio de Janeiro: Instituto Brasileiro de Geografia e Estatística; 2014.

10. Instituto Brasileiro de Geografia e Estatística. Pesquisa Nacional de Saúde do Escolar(PeNSE), 2012. Rio de Janeiro: Instituto Brasileiro de Geografia e Estatística; 2013.

11. Cleland VJ, Schmidt MD, Dwyer T, Venn AJ. Television viewing and abdominal obesity in young adults: is the association mediated by food and beverage consumption during viewing time or reduced leisure-time physical activity? Am J Clin Nutr 2008; 87:1148-55.

12. Braithwaite I, Stewart AW, Hancox RJ, Beasley R, Murphy R, Mitchell EA. The worldwide association between television viewing and obesity in children and adolescents: cross sectional study. PLoS One 2013; 8:e74263.

13. Poterico JA, Bernabé-Ortiz A, Mola CL, Miranda JJ. Association between television viewing and obesity in Peruvian women. Rev Saúde Pública 2012; 46:610-6. 
14. Steffen LM, Dai S, Fulton JE, Labarthe DR. Overweight in children and adolescents associated with TV viewing and parental weight: Project Heart Beat! Am J Prev Med 2009; 37(1 Suppl):S50-5.

15. Swinburn B, Shelly A. Effects of TV time and other sedentary pursuit. Int J Obes (Lond) 2008; 32 Suppl 7:S132-6.

16. Hu FB, Li TY, Colditz GA, Willett WC, Manson JE. Television watching and other sedentary behaviors in relation to risk of obesity and type 2 diabetes mellitus in women. JAMA 2003; 289:1785-91.

17. Dunstan DW, Salmon J, Owen N, Armstrong T, Zimmet PZ, Welborn TA, et al. Associations of TV viewing and physical activity with the metabolic syndrome in Australian adults. Diabetologia 2005; 48:2254-61.

18. Dunstan DW, Barr ELM, Healy GN, Salmon J, Shaw JE, Balkau B, et al. Television viewing time and mortality. Circulation 2010; 121: 384-91.

19. Wijndaele K, Brage S, Besson H, Khaw KT, Sharp SJ, Luben R, et al. Television viewing time independently predicts all-cause and cardiovascular mortality: the EPIC Norfolk Study. Int J Epidemiol 2010; 40:150-9.

20. Inoue S, Sugiyama T, Takamiya T, Oka K, Owen N, Shimomitsu T. Television viewing time is associated with overweight/obesity among older adults, independent of meeting physi$\mathrm{cal}$ activity and health guidelines. J Epidemiol 2012; 22:50-6.

21. Harris JL, Sarda V, Schwartz MB, Brownell KD. Redefining "child-directed advertising" to reduce unhealthy television food advertising. Am J Prev Med 2013; 44:358-64.

22. Almeida SS, Nascimento PCBD, Quaioti TCB. Quantidade e qualidade de produtos alimentícios anunciados na televisão brasileira. Rev Saúde Pública 2002; 36:353-5.

23. Harris JL, Bargh JA, Brownell KD. Priming effects of television food advertising on eating behavior. Health Psychol 2009; 28:404-13.

24. Costa MLG, Oliveira PAD, Auad SM. Publicidade de alimentos para o público infantil na televisão e diretrizes alimentares brasileiras: sintonia ou confronto? Arq Odontol 2011; 47:181-7.

25. Marins BR, Araújo IS, Jacob SC. A propaganda de alimentos: orientação, ou apenas estímulo ao consumo? Ciênc Saúde Coletiva 2011; 16:3873-82.

26. Costa SMM, Horta PM, Santos LC. Análise dos alimentos anunciados durante a programação infantil em emissoras de canal aberto no Brasil. Rev Bras Epidemiol 2013; 16:97683.

27. Departamento de Atenção Básica, Secretaria de Atenção à Saúde, Ministério da Saúde. Guia alimentar para a população brasileira. 2a Ed. Brasília: Ministério da Saúde; 2014.
28. Kelly B, King L, Baur L, Rayner M, Lobstein T, Monteiro C, et al. Monitoring food and nonalcoholic beverage promotions to children. Obes Rev 2013; 14:59-69.

29. Keller SK, Schulz PJ. Distorted food pyramid in kids programmes: a content analysis of television advertising watched in Switzerland. Eur J Public Health 2010; 21:300-5.

30. Kelly B, Halford JCG, Boyland EJ, Chapman $\mathrm{K}$, Bautista-Castaño I, Berg C, et al. Television food adverting to children: a global perspective. Research and practice. Am J Public Health 2010; 100:1730-6.

31. Effertz T, Wilcke AC. Do television food commercials target children in Germany? Public Health Nutr 2012; 15:1466-73.

32. Guran T, Turan S, Akcay T, Degirmenci F, Avci $\mathrm{O}$, Asan A, et al. Content analysis of food advertising in Turkish television. J Pediatr Child Health 2010; 46:427-30.

33. Harris JL, Bargh JA, Brownell KD. Priming effects of television food advertising on eating behavior. Health Psychol 2009; 28:404-13.

34. Scully M, Dixon H, Wakefield M. Association between commercial television exposure and fast-food consumption among adults. Public Health Nutr 2009; 12:105-10.

35. Agência Nacional de Vigilância Sanitária. RDC no 24, 2010. Dispõe sobre a oferta, propaganda, publicidade, informação e outras práticas correlatas cujo objetivo seja a divulgação e a promoção comercial de alimentos considerados com quantidades elevadas de açúcar, de gordura saturada, de gordura trans, de sódio, e de bebidas com baixo teor nutricional. http:// 189.28.128.100/nutricao/docs/legislacao/re solucao_rdc24_29_06_2010.pdf (acessado em 30/Nov/2015).

36. Gomes FS, Castro IRR, Monteiro CA. Publicidade de alimentos no Brasil: avanços e desafios. Ciênc Cult (São Paulo) 2010; 62:48-51.

37. Brasil. Lei no 8.078, de 11 de setembro de 1990. Dispõe sobre a proteção do consumidor e dá outras providências. Diário Oficial da União 1990; 12 set.

38. Conselho Nacional dos Direitos da Criança e do Adolescente. Resolução no 163, de 13 de março de 2014. Dispõe sobre a abusividade do direcionamento de publicidade e de comunicação mercadológica à criança e ao adolescente. Diário Oficial da União 2014; 4 abr.

39. Decisão do STJ define publicidade voltada à criança como "abusiva". O Estado de S. Paulo 2016; 10 mar. http://economia.estadao.com. br/noticias/geral,decisao-do-stj-define-publi cidade-voltada-a-criancas-como-abusiva, 10000020623 (acessado em 20/Mar/2016).

40. Koordeman R, Anschutz DJ, van Baaren RB, Engels RCME. Exposure to soda commercials affects sugar-sweetened soda consumption in young women: an observational experimental study. Appetite 2010; 54:619-22. 
41. Anschutz DJ, Engels RCME, van der Zwaluw CS, van Strien T. Sex differences in young adults' snack food intake after food commercial exposure. Appetite 2011; 56:255-60.

42. Utter J, Scragg R, Schaaf D. Associations between television viewing and consumption of commonly advertised foods among New Zealand children and young adolescents. Public Health Nutr 2006; 9:606-12.

43. Boyland EJ, Harrold JA, Kirkham TC, Corker C, Cuddy J, Evans D, et al. Food commercials increase preference for energy-dense foods, particulary in children who watch more television. Pediatrics 2011; 128:e93-100.

44. Dixon HG, Scully ML, Wakefield MA, White VM, Crawford DA. The effects of television advertisements for junk food versus nutritious food on children's food attitudes and preferences. Soc Sci Med 2007; 65:1311-23.
45. Tapper K, Jiga-Boy G, Maio GR, Haddock G Lewis M. Development and Preliminary evaluation of an internet-based healthy eating program: randomized controlled trial. J Med Internet Res 2014; 16:e231.

46. Elliot C, Brierley M. Health choice: exploring how children evaluate the healthfulness of packaged foods. Can J Public Health 2012; 103:e453-8

47. Veerman JL, van Beeck EF, Barendregt JJ, Mackenbach JP. By how much would limiting TV food advertising reduce childhood obesity? Eur J Public Health 2009; 19:365-9. 


\section{Abstract}

This study aimed to analyze TV food advertising in Brazil based on the recommendations of the Food Guide for the Brazilian Population, 2014. Programming from the four most popular $T V$ channels was recorded on two non-consecutive days in January 2014. The commercials were categorized in (i) foods and beverages, (ii) restaurants, and (iii) non-food services, goods, and products, with those in the first category subdivided according to recommendations in the food guide. The commercials' categories were described by means of their frequency (and 95\% confidence interval), for all the records and according to the day of the week. Of the 2,732 commercials that were identified, food and beverage advertising was the third largest category, with $10.2 \%$ of the total. In this category, ultra-processed foods accounted for $60.7 \%$ of the commercials, while fresh or minimally processed foods at around $7 \%$. The findings run counter to the guide's recommendations, reinforcing the importance of measures to regulate food advertising in Brazil.

Food Publicity; Television; Chronic Disease

\section{Resumen}

El objetivo del estudio es analizar la publicidad televisiva de alimentos en Brasil, en base a las recomendaciones de la Guía Alimentaria para la Población Brasileña de 2014. La programación de los cuatro canales más populares de televisión fue grabada en enero de 2014, durante dos días no consecutivos. Los anuncios fueron categorizados en (i) alimentos $y$ bebidas, (ii) restaurantes $y$ (iii) servicios, bienes y productos no alimenticios, siendo aquellos referentes a la alimentación subdivididos conforme las recomendaciones de la guía. Las categorías de anuncios fueron descritas mediante su frecuencia (e intervalo de 95\% de confianza), para el conjunto total de los registros y según el día de la semana. De los 2.732 anuncios que se identificaron, la publicidad de alimentos y bebidas representó la tercera mayor categoría anunciada, con un 10,2\%. En esa categoría, los alimentos ultraprocesados correspondieron a un 60,7\% de los anuncios, $y$ los alimentos in natura o mínimamente procesados a cerca de un 7\%. La realidad evidenciada se opone a las recomendaciones de la guía, reforzando la importancia de acciones para la regulación de la publicidad de alimentos en Brasil.

Publicidad de Alimentos; Televisión; Enfermedad Crónica
Recebido em 17/Dez/2015

Versão final reapresentada em 31/Mai/2016

Aprovado em 24/Jun/2016 